\title{
Padrões de herbivoria e estratégias de defesa de comunidades de restinga em gradiente edáfico
}

\author{
(1) Fernanda Vitorio da Silva ${ }^{1}$, João Carlos Ferreira de Melo Júnior ${ }^{1,3}$ e ${ }^{\text {Maiara Matilde-Silva }}{ }^{2}$
}

Como citar: Silva, F.V., Melo Júnior, J.C.F. \& Matilde-Silva, M. 2021. Padrões de herbivoria e estratégias de defesa de comunidades de restinga em gradiente edáfico. Hoehnea 49: e212021. https://doi.org/10.1590/2236-8906-21/2021

\begin{abstract}
Herbivory patterns and defense strategy of Restinga communities in edaphic gradients) This study aimed to evaluate the spatial distribution of defenses and herbivory rates in 20 plant species and their relationship with the edaphic gradient in four sandbank formations in the State Park of Acaraí (PEA) in Santa Catarina State, Brazil. The morphoanatomical, histochemical and nutritional attributes of the leaves were measured on twenty leaves of ten individuals of each species. Six soil samples were collected in each formation for analysis of macronutrient contents, organic matter, moisture and litter thickness. Statistical analyzes were performed with the R software. The results indicated that herbivory rates vary according to leaf morphoanatomical and histochemical attributes, which respond to the soil's nutritional and water characteristics and, therefore, influence the anti-herbivory groups in each plant community.
\end{abstract}

Keywords: edaphic quality, environmental filter, plant-herbivore interaction, plant-soil relationship

RESUMO - (Padrões de herbivoria e estratégias de defesa de comunidades de restinga em gradiente edáfico) O estudo objetivou avaliar a distribuição espacial das defesas e das taxas de herbivoria em 20 espécies vegetais e a relação destas com o gradiente edáfico em quatro formações de restinga no Parque Estadual do Acaraí (PEA), Estado de Santa Catarina, Brasil. Atributos morfoanatômicos, histoquímicos e nutricionais foliares foram medidos em vinte folhas de dez indivíduos de cada espécie. Seis amostras de solo foram coletadas em cada formação para análise dos teores de macronutrientes, matéria orgânica, umidade e espessura da serapilheira. As análises estatísticas foram realizadas utilizando software R. Os resultados indicaram que as taxas de herbivoria variam de acordo com os atributos morfoanatômicos e histoquímicos foliares, os quais respondem às características nutricionais e hídricas do solo, influenciando o agrupamento de defesas anti-herbivoria em cada comunidade vegetal.

Palavras-chave: filtro ambiental, interação planta-herbívoro, qualidade edáfica, relação planta-solo

\section{Introdução}

Plantas e insetos herbívoros têm coexistido por, aproximadamente, 350 milhões de anos, e tal interação influencia diversos processos ecológicos e evolutivos nos ecossistemas terrestres e aquáticos (Gatehouse 2002). Diante de recursos com diferentes características nutricionais e palatabilidade, os herbívoros desenvolveram diversas adaptações, a fim de maximizar o uso dos recursos alimentares; e as plantas, por sua vez, produziram uma diversidade de estratégias para reduzir o impacto dos herbívoros.

As estratégias de defesa contra a herbivoria constituemse em: i) tolerância à herbivoria, onde os danos causados à planta não interferem no crescimento e desempenho; e ii) resistência, representando a produção de defesas que visam evitar ou impedir a herbivoria (Mauricio 2000). As defesas físicas são atributos morfológicos ou anatômicos, tais como: tricomas tectores ou glandulares, espinhos, acúleos, cutícula espessa, laticíferos, epiderme espessa, hipoderme, desenvolvimento de tecidos mecânicos, e inclusões minerais
(Hanley et al. 2007). As defesas químicas compreendem os metabólitos secundários tóxicos ou que repelem os herbívoros (Hartman 2004). Estes são classificados de acordo com a sua composição química em: compostos fenólicos; terpenóides; e compostos nitrogenados (Kabera et al. 2014). Defesas nutricionais influenciam a preferência alimentar através da redução da absorção dos nutrientes pelos herbívoros (Pais 1998), pois ingerir folhas com baixo teor de nitrogênio pode afetar fatores associados à manutenção da vida do inseto, assim como, por exemplo, limitar o crescimento e a fecundidade, além de alterar as taxas de mortalidade (Howe \& Schaller 2008).

Tais características defensivas podem estar sob pressões seletivas por duas vias: top-down, que se refere à pressão causada pelos herbívoros que leva a mudanças na expressão de defesas; e botom-up que refere-se à qualidade do ambiente em relação com a produção de defesas (Agrawal \& Fishein 2006, Loiola et al. 2012). Neste sentido, as taxas de consumo e a extensão do dano foliar provocado às plantas são controlados pelos atributos de defesa, os quais podem ser influenciados pela qualidade do ambiente

\footnotetext{
1. Universidade da Região de Joinville, Departamento de Ciências Biológicas, Laboratório de Morfologia e Ecologia Vegetal, Rua Paulo Malschitzki, 10, Zona Industrial 89219-710 Joinville, SC, Brasil

2. Universidade Federal do Paraná, Departamento de Ecologia, Setor de Ciências Biológicas, Avenida Cel. Francisco H. dos Santos, 100 , Jardim das Américas, 89530-000, Curitiba, PR, Brasil

3. Autor para correspondência: joao.melo@univille.br
} 
(Espírito-Santo et al. 2007). Diversos estudos mostram que a disponibilidade de nutrientes no solo afeta a qualidade das plantas e a relação planta-inseto (Root 1972, Marques et al. 2000, Neves et al. 2010). Solos pobres em nutrientes possuem precária disponibilidade de minerais, tais como N, P, Ca e $\mathrm{K}$, os quais limitam o crescimento das plantas, levando ao acúmulo de carbono e a um aumento geral na proporção da parede celular (Gonçalves-Alvim et al. 2006). Sobre tais solos, as folhas das plantas tornam-se espessas, resistentes e rígidas, uma condição denominada "esclerofilia" (Turner 1994). Por outro lado, plantas que ocorrem em áreas com maior disponibilidade de recursos nutricionais e hídricos investem pouca energia na produção de defesas (Coley et al. 1985). Em compensação, apresentam alta taxa de expansão foliar, o que diminui o tempo de vulnerabilidade à herbivoria (Kursar \& Coley 2003).

Tais variações edáficas podem ser encontradas no ecossistema de restinga, ambiente localizado na planície litorânea da costa brasileira (Melo Jr. \& Boeger 2015). Formado por sedimentos de origem marinha (Sampaio et al. 2005), os solos sob vegetação de restinga, são arenosos e quimicamente pobres, tendo como principal fonte de nutrientes a decomposição de serapilheira e o spray marinho (Leão \& Dominguez 2000). Desta forma, o conjunto de comunidades vegetais ali instaladas está sujeito a condições ambientais mais extremas do que aquelas encontradas nos demais ecossistemas e formações do bioma Mata Atlântica (Scarano et al. 2001, Scarano 2002).

Melo Jr \& Boeger (2015) evidenciaram que há um gradiente edáfico correlacionado com a diversidade e a estrutura das formações de restinga. Variáveis edáficas, tais como teores de matéria orgânica, acidez potencial e a espessura da serapilheira ao longo do gradiente, são atributos de maior influência sobre a distribuição das espécies e o padrão estrutural das comunidades vegetais.

A dissimilaridade nutricional ao longo do gradiente edáfico, no sentido mar-continente, causa acentuada substituição de espécies vegetais ao longo de todas as formações da restinga (Melo \& Boeger 2015). Tais variações no solo atuam como filtro ambiental para a seleção de espécies adaptadas às características edáficas de cada formação vegetal (Fukami et al. 2005), influenciando na expressão de defesas nas espécies. As estratégias defensivas devem se apresentar agrupadas devido às similaridades do ambiente, podendo atuar como grupos funcionais de defesas (Silva \& Batalha 2011) e, por conseguinte, influenciar a composição da entomofauna.

Diante do exposto, o estudo objetiva investigar como a variação espacial na qualidade edáfica atua sobre a produção de defesas em plantas de restinga, e como tais atributos regulam os danos por herbivoria ao longo do gradiente edáfico. As hipóteses do estudo são: i) a intensidade das defesas é decrescente no sentido mar-continente; ii) as espécies ocorrentes na restingas herbácea e arbustiva apresentam alto investimento em defesas estruturais e compostos fenólicos (defesas baseadas em carbono); iii) a taxa de herbivoria é maior nas espécies ocorrentes na floresta de transição.

\section{Material e Métodos}

A área de estudo está localizada no Parque Estadual do Acaraí (PEA) (figura 1), Unidade de Conservação de Proteção Integral do município de São Francisco do Sul, litoral nordeste do estado de Santa Catarina (26 ${ }^{\circ} 30^{\prime} \mathrm{S}$ e $\left.40^{\circ} 37^{\prime} \mathrm{W}\right)$. O clima, fortemente influenciado pela umidade marítima, é mesotérmico com verões quentes, sem déficit hídrico, segundo Köppen e Geiger a classificação do clima é Cfa, com temperatura média anual de $21.2^{\circ} \mathrm{C}$ e índices pluviométricos médios de $1.830 \mathrm{~mm}$ ao ano (Knie 2002). O parque compõe um local de alta diversidade (Melo Jr. \& Boeger 2015) e importância ecológica, é classificado como de prioridade alta no Projeto de Conservação e Utilização Sustentável da Diversidade Biológica Brasileira (Probio 2013).

A restinga herbácea $(\mathrm{Rh})$, composta por plantas psamófilas e halófitas, ocorre sobre Neossolo Quartzarênico com baixa capacidade de retenção de água e nutrientes no solo e baixa fertilidade. A restinga arbustiva (Ra) é formada por um denso mosaico de arbustos sobre Espodossolo Ferrihumilúvico de natureza não hidromórfica e com baixa fertilidade. A restinga arbustivo-arbórea (Raa) é composta por arbustos e árvores, com condições edáficas semelhantes a Ra. A floresta de transição $(\mathrm{Ft})$ é caracterizada por um estrato arbóreo contínuo sobre Espodossolo Ferrihumilúvico + Organossolo Hálico de natureza hidromórfica (Melo Jr. \& Boeger 2015). O gradiente edáfico apresenta baixo teor de matéria orgânica e baixa retenção de água nas formações mais próximas ao mar, com níveis crescentes desses fatores em direção à floresta.

Foram utilizadas para estudo cinco espécies (tabela 1) com maior índice de importância em cada fitofisionomia da restinga do PEA(Melo Júnior \& Boeger 2015), totalizando 20 espécies vegetais para as quatro formações. Dez indivíduos adultos de cada espécie foram selecionados aleatoriamente. Foram coletadas 20 folhas maduras de cada indivíduo, inseridas do $3^{\circ}$ ao $6^{\circ}$ nó, das quais cinco folhas foram fixadas em solução de Karnovisk (paraformaldeído + glutaraldepido) por 72 horas e, posteriormente, conservadas em álcool $70 \%$ para testes histoquímicos e 15 folhas foram secas em estufa de circulação de ar forçado por 72 horas, para posterior morfometria.

Foram analisados os atributos morfológicos: massas fresca e seca $(\mathrm{g})$ em balança analítica de precisão para estimar a quantidade de água presente na folha; áreas foliares completa e restante $\left(\mathrm{cm}^{2}\right)$, após herbivoria, medidas com software Sigma Scan PRO (version 5.0, SPSS Inc., Chicago IL, USA), para, posteriormente estimar a área foliar específica $\left(\mathrm{cm}^{2} / \mathrm{g}\right)$, e a porcentagem de herbivoria (área completa/área restante*100). Para o cálculo da razão de herbivoria foram consideradas somente as folhas herbivoradas.

Utilizando lâmina de aço, foram produzidas secções transversais das folhas previamente fixadas em Karnovisk. Foram aplicadas sobre as secções, as soluções de reagente de Dragendorf para compostos alcalóides (Johansen 1940), soluções aquosas de cloreto férrico $10 \%$ para testar a presença de compostos fenólicos (Johansen 1940), vanilina clorídrica 


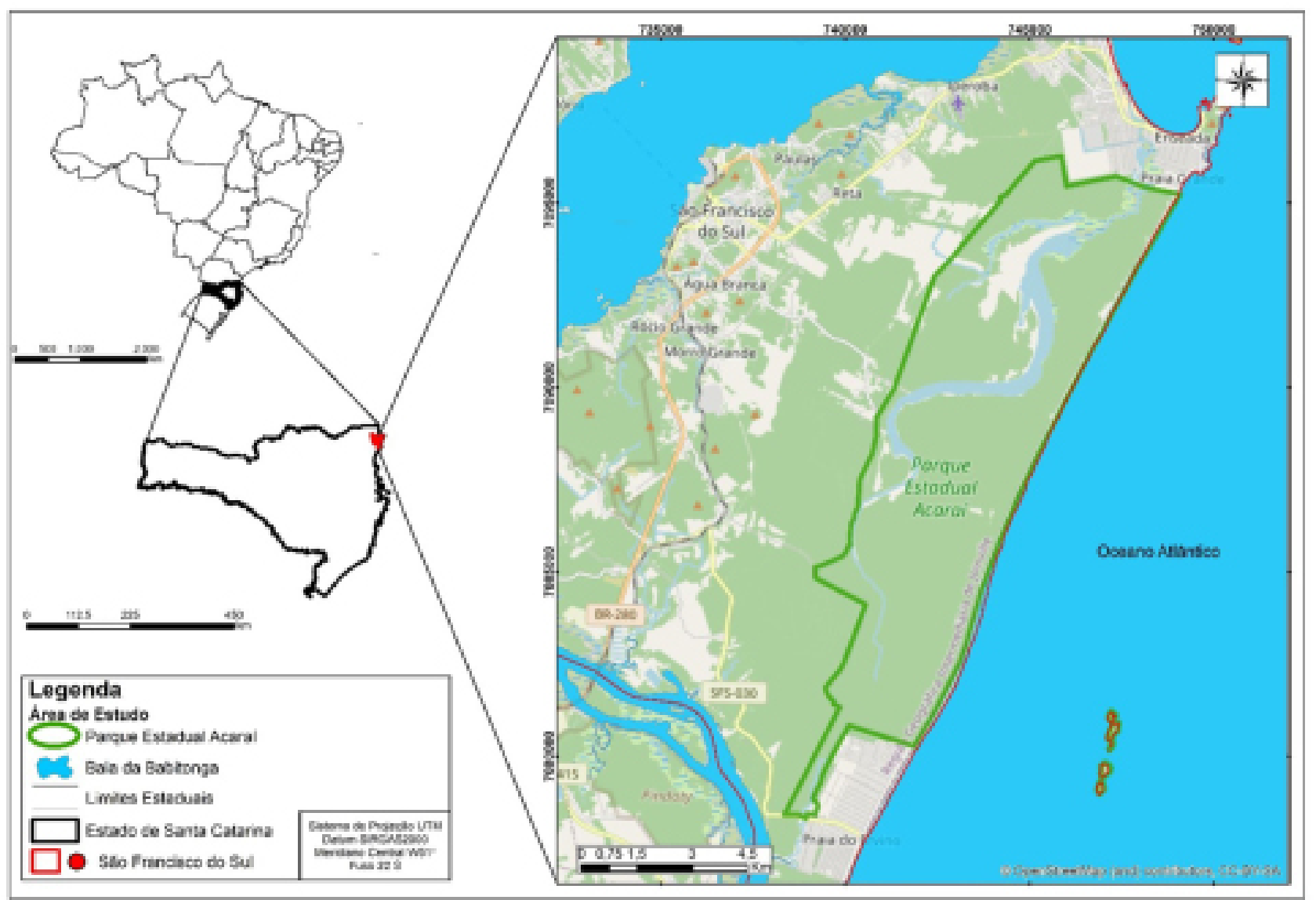

Figura 1. Localização da área de estudo: Parque Estadual do Acaraí (PEA) no município de São Francisco do Sul, Estado de Santa Catarina, Brasil.

Figure 1. Location of the study area: Parque Estadual do Acaraí (PEA) in the municipality of São Francisco do Sul, Santa Catarina State, Brazil.

Tabela 1: Espécies amostradas nas formações de restinga do Parque Estadual Acaraí, São Francisco do Sul, Estado de Santa Catarina, Brasil. No Col.: Número do coletor JCMelo.

Table 1: Species sampled in the sandbank formations of the Parque Estadual Acaraí, São Francisco do Sul, Santa Catarina State, Brazil. N ${ }^{\circ}$ Col.: Collector number JCMelo.

\begin{tabular}{|c|c|c|c|}
\hline Formação & Espécie & Família & $\mathrm{N}^{\mathrm{o}}$ Col. \\
\hline \multirow{5}{*}{$\begin{array}{l}\text { Restinga } \\
\text { herbácea }\end{array}$} & Smilax campestris Griseb. & Smilacaceae & 1018 \\
\hline & Ipomoea imperati (Vahl) Griseb & Convolvulaceae & 1003 \\
\hline & Canavalia rósea (Sw.) DC. & Fabaceae & 1000 \\
\hline & Scaevola plumieri (L.) Vahl & Goodeniaceae & 1101 \\
\hline & Ipomoea pes-caprae (L.) R. Br & Convolvulaceae & 1032 \\
\hline \multirow{5}{*}{$\begin{array}{l}\text { Restinga } \\
\text { arbustiva }\end{array}$} & Guapira opposita (Vell.) Reitz & Nyctaginaceae & 1098 \\
\hline & Myrcia pulchra (O.Berg) Kiaersk. & Myrtaceae & 1060 \\
\hline & Shcwartzia brasiliense (Choisy) Bedell ex Gir-Cañas & Marcgraviaceae & 1087 \\
\hline & Varronia curassavica (Jacq.) Roem. \& Schult. & Boraginaceae & 1013 \\
\hline & Clusia criuva Cambess. & Clusiaceae & 1078 \\
\hline \multirow{5}{*}{$\begin{array}{l}\text { Restinga } \\
\text { arbustivo- } \\
\text { arbórea }\end{array}$} & Pera glabrata Poepp. ex Baill. & Peraceae & 1152 \\
\hline & Ocotea pulchella (Nees \& Mart.) Mez & Lauraceae & 1085 \\
\hline & Ternstroemia brasiliensis Cambess. & Pentaphylacaceae & 1074 \\
\hline & Myrsine venosa A.DC. & Primulaceae & 1129 \\
\hline & Ilex theezans Mart. ex Reissek & Aquifoliaceae & 1073 \\
\hline \multirow{5}{*}{$\begin{array}{l}\text { Floresta de } \\
\text { transição }\end{array}$} & Calophyllum brasiliense Camb. & Calophyllaceae & 1072 \\
\hline & Andira fraxinifolia Benth. & Fabaceae & 1126 \\
\hline & Picramnia parviflora Engl. & Simaroubaceae & 1268 \\
\hline & Alchornea triplinervia (Spreng.) Müll. Arg. & Euphorbiaceae & 1164 \\
\hline & Nectandra oppositifolia Nees \& Mart. ex Nees & Lauraceae & 1101 \\
\hline
\end{tabular}


1\% para testar a presença de tanino (Burns 1971), reagente de Nadi para avaliação de terpenos (David \& Carde 1964). Teste branco, sem adição de reagentes, foi produzido para fins de comparação. As secções foram montadas entre lâmina e lamínula com água glicerinada $30 \%$ e vedadas com Verniz Vitral incolor (Paiva et al. 2006). Para fins de distinção de estruturas, foi considerado como cutícula a deposição de cutina (cuticularização) sobre a face externa da epiderme da lâmina foliar (Barthlott et al. 1998).

Para análise de teor de nitrogênio, as folhas foram secas a uma temperatura média constante de $47^{\circ} \mathrm{C}$, passadas em moinho de bola a granulometria de $0,25 \mathrm{~mm}$ e, utilizando 0,20 gramas do material moído foi estimado o teor de nitrogênio pelo método Kjeldahl (Yasuhara \& Nokihara 2001).

Foram coletadas seis amostras de solo em cada uma das quatro fitofisionomias estudadas, com profundidade de 15 $\mathrm{cm}$ para a análise dos teores de macronutrientes e umidade gravimétrica após homogeneização. Em laboratório, uma fração do solo foi pesada em balança analítica de precisão e, após 72 horas de secagem em estufa a $105^{\circ} \mathrm{C}$, foi novamente pesada para estimar a disponibilidade hídrica do solo em cada formação pelo método da umidade gravimétrica (Santos et al. 2013). Segundo os métodos descritos em Tedesco et al. (1995), foi realizada a homogeneização das amostras para a produção de três amostras compostas por formação de restinga destinada a uma avaliação química do solo por meio das variáveis de $\mathrm{pH}$, fósforo $(\mathrm{P})$, potássio $(\mathrm{K})$, sódio $(\mathrm{Na})$, magnésio $(\mathrm{Mg})$, acidez potencial $(\mathrm{H}+\mathrm{Al}$, íons $\mathrm{H}+\mathrm{e}$ $\left.\mathrm{Al}^{3}+\right)$, soma de bases (SB), capacidade de troca catiônica (CTC), saturação por bases (V) e matéria orgânica (MO) no Laboratório de Análise Química de Solo da EPAGRI/SC.

Para todos os atributos biológicos e de solo foram calculadas as médias e os respectivos desvios-padrão. Os valores médios foram submetidos à análise de variância (ANOVA one-way) e os atributos de solo à uma análise de componentes principais (PCA) para verificar quais variáveis promovem maior diferenciação entre as formações de restinga. Os testes estatísticos foram realizados com software R com nível de significância de $\alpha=5 \%$ (Crawley 2007).

\section{Resultados}

Foram verificadas diferenças estatisticamente significantes nos atributos morfológicos foliares avaliados e taxas de herbivoria entre as comunidades estudadas.

A área específica foliar (AEF) apresentou padrão de aumento no sentido mar-continente, exceto pela restinga arbustivo-arbórea, que apresentou o menor valor de AEF. A área foliar (AF) foi similar entre as restingas herbácea e arbustivo-arbórea, enquanto a menor e maior áreas foliares ocorreram na restinga herbácea e floresta de transição, respectivamente. Os atributos grau de suculência e teor de nitrogênio apresentaram padrão decrescente da restinga herbácea para a floresta de transição. As massas seca e fresca foram maiores na restinga herbácea, seguidos da restinga arbustiva. A restinga arbustivo-arbórea e floresta de transição apresentaram valores similares de massas seca e fresca. A maior força de perfuração ocorreu na restinga arbustivoarbórea e a menor na restinga arbustiva. Nos dois extremos do gradiente, na restinga herbácea e na floresta de transição, a força de perfuração foi mediana e similar (tabela 2).

A taxa de herbivoria apresentou padrão de diminuição ao longo do gradiente ambiental estudado, sendo a maior taxa de herbivoria na restinga herbácea e a menor na floresta de transição (tabela 2).

A análise histoquímica evidenciou maior presença de metabólitos secundários nos tecidos foliares das populações de restinga arbustivo-arbórea e floresta de transição. De modo geral, as faces abaxial e adaxial da epiderme e os parênquimas clorofilianos apresentaram alto grau de proteção por metabólitos secundários, com destaque para os terpenos e taninos. As espécies da restinga herbácea apresentaram menor presença de metabólitos secundários, sendo mais protegidas por terpenos, em comparação com alcaloides e taninos (tabela 3 ).

A análise de componentes principais (PCA) explicou $79 \%$ da variação dos dados de solo. $46 \%$ foi explicado pelos atributos de $\mathrm{pH}$ e MO relacionados ao eixo 1 e $33 \%$ relacionados aos atributos de serapilheira e umidade no eixo 2 (tabela 4, figura2). Isso significa que essas quatro variáveis edáficas é que promoveram maior diferenciação entre as formações da restinga.

A floresta de transição apresentou composição química de solo que divergiu expressivamente das demais formações, indicando solo mais nutritivo. Os teores de matéria orgânica, potássio, alumínio, espessura da serapilheira e umidade gravimétrica no solo aumentaram, gradativamente, da restinga herbácea em direção à floresta. Os teores de sódio, fósforo e cálcio, a soma de bases, a saturação por bases e a capacidade de troca cationica foram menores na restinga arbustivo-arbórea, similares entre as restingas herbácea e arbustiva e aumentaram abruptamente na floresta. $\mathrm{O}$ grau de acidez do solo mostrou-se crescente no sentido marcontinente, porém tornou-se levemente menos ácido no ambiente florestal (tabela 4).

\section{Discussão}

Estratégias anti-herbivoria - A disponibilidade hídrica, de nutrientes e de metabólitos secundários foram os atributos foliares que melhor explicaram a variação das taxas de herbivoria entre as quatro formações de restinga do PEA.

As nossas primeira e segunda hipóteses não foram corroboradas, uma vez que as defesas não apresentaram padrão unidirecional (redução da intensidade de defesas no sentido mar-continente). Diferindo do esperado, a dureza foliar foi similar entre as comunidades de restinga herbácea, arbustiva e florestal. Além disso, diferindo do pressuposto da segunda hipótese, as comunidades de restinga arbustivo-arbórea e da floresta de transição apresentaram maior investimento em taninos (compostos fenólicos) em comparação à comunidade de restinga herbácea.

Nas plantas da formação herbácea foram observados os maiores teores de nitrogênio e de disponibilidade hídrica. Tais atributos, aliados à pouca defesa química nas plantas desta formação, resultou em maiores danos causados por insetos herbívoros. Moran \& Hamilton (1980), Furlan et al. (1999) e Coley \& Barone (1996) mostraram que espécies com maiores quantidades de nitrogênio nas folhas poderiam ter 


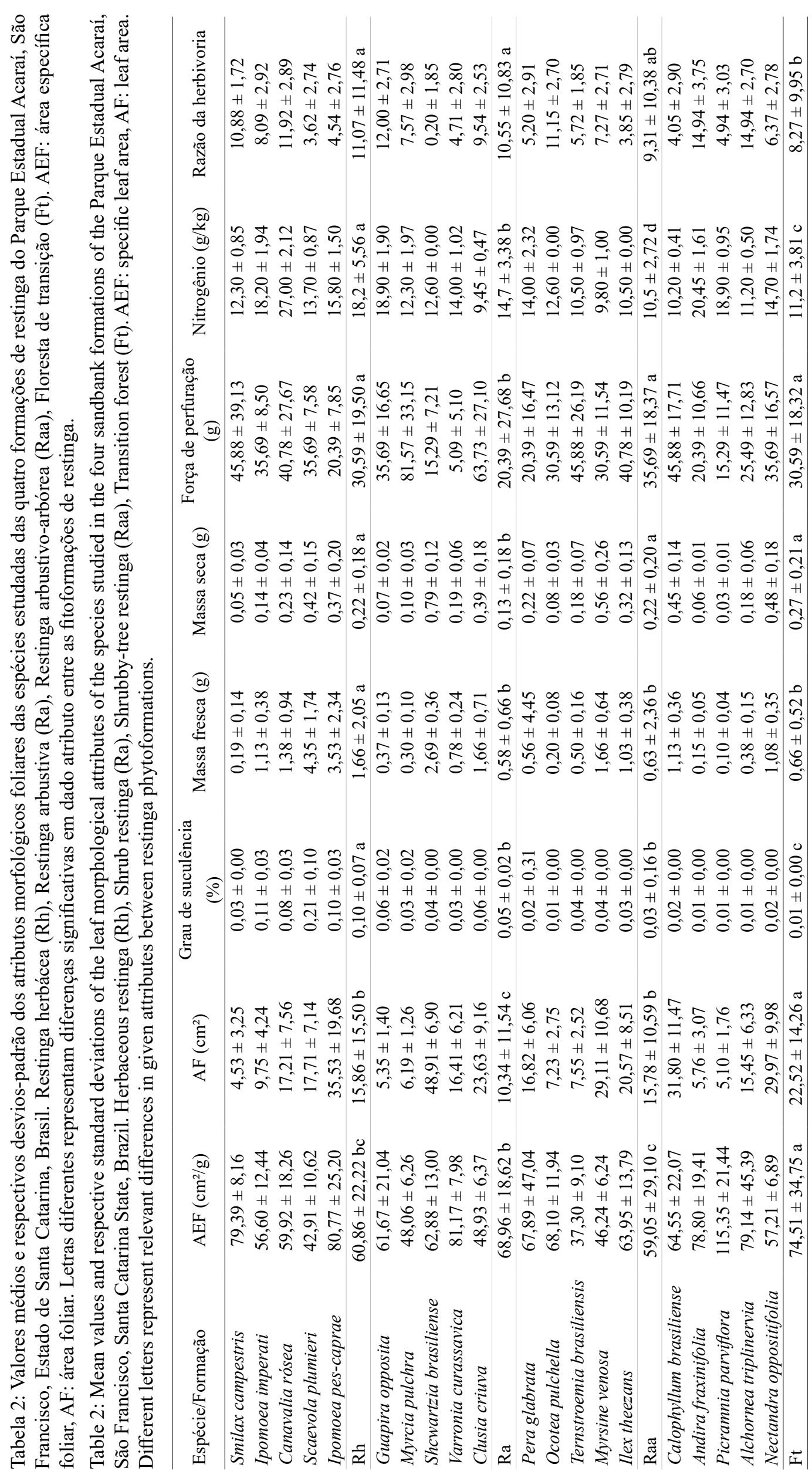




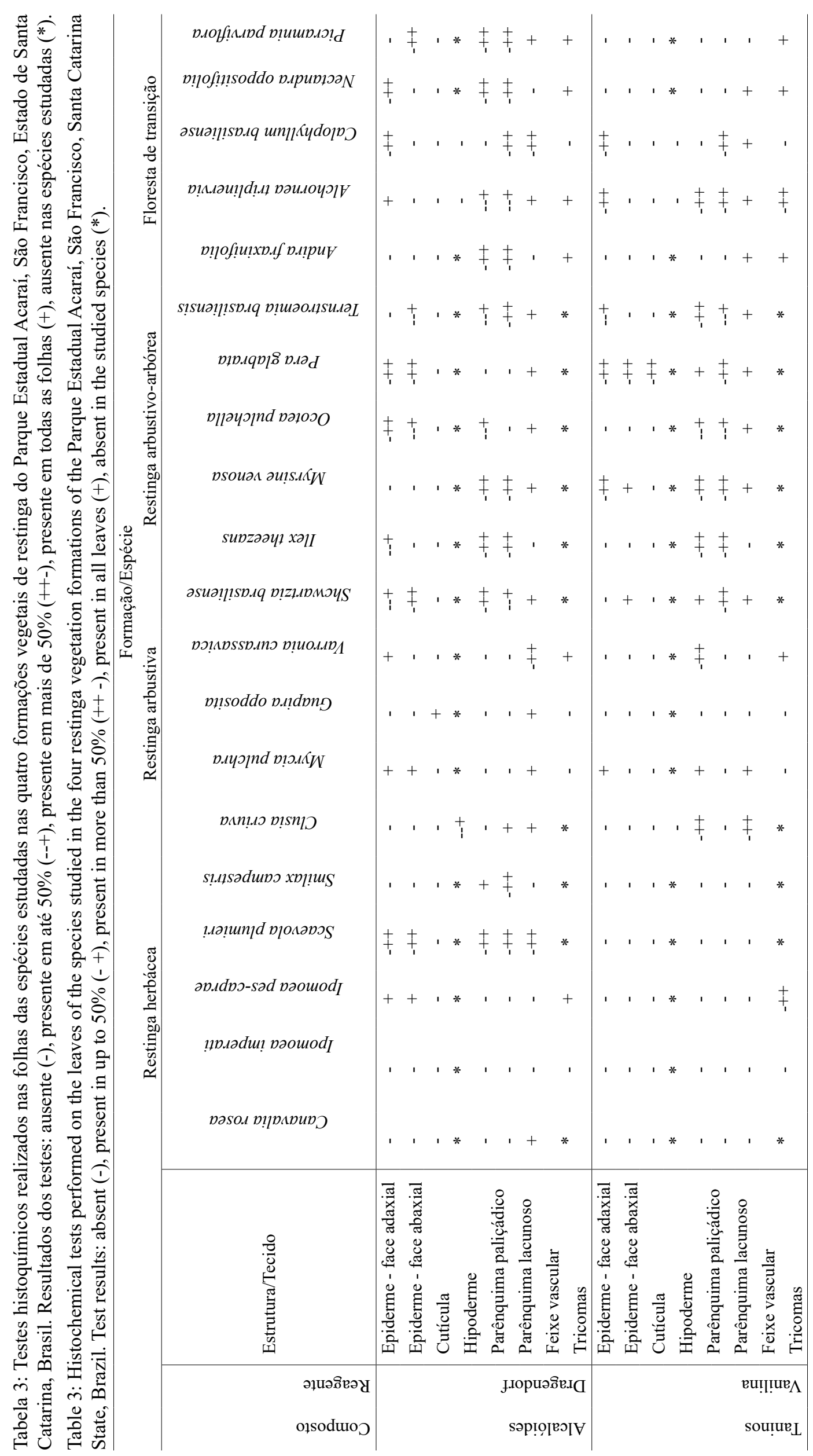


Tabela 4: Valores médios dos atributos nutricionais do solo, nas quatro formações vegetais de restinga do Parque Estadual Acaraí, São Francisco, Estado de Santa Catarina, Brasil. SB: Soma de bases, CTC: Capacidade de troca catiônica, V: Saturação por bases, MO: Matéria orgânica. Formação: Restinga Herbácea (Rh), Restinga arbustiva (Ra), Restinga arbustivo-arbórea (Raa), Floresta de transição (Ft).

Table 4: Mean values of the nutritional attributes of the soil, in the four restinga vegetation formations of the Parque Estadual Acaraí, São Francisco, Santa Catarina State, Brazil. SB: Sum of bases, CTC: Capacity of cation exchange, V: Saturation by bases, MO: Organic matter. Formation: Restinga Herbácea (Rh), Restinga arbustiva (Ra), Restinga arbustivo-arbórea (Raa), Transition forest (Ft).

\begin{tabular}{|c|c|c|c|c|c|c|}
\hline \multirow{2}{*}{ Atributos ambientais } & \multicolumn{4}{|c|}{ Formações de restinga } & \multicolumn{2}{|c|}{ PCA } \\
\hline & $\mathrm{Rh}$ & $\mathrm{Ra}$ & Raa & $\mathrm{Ft}$ & Eixo 1 & Eixo 2 \\
\hline $\mathrm{MO}\left(\mathrm{g} / \mathrm{dm}^{3}\right)$ & 3,72 & 7,30 & 7,59 & 43,14 & 0,35 & 0,22 \\
\hline $\mathrm{pH}$ & 5,49 & 5,02 & 4,42 & 5,35 & $-0,36$ & 0,14 \\
\hline $\mathrm{P}\left(\mathrm{mg} / \mathrm{dm}^{3}\right)$ & 9,24 & 6,60 & 1,05 & 110,64 & $-0,21$ & 0,40 \\
\hline $\mathrm{K}\left(\mathrm{cmolc} / \mathrm{dm}^{3}\right)$ & 6,57 & 13,67 & 14,65 & 23,14 & $-0,34$ & 0,21 \\
\hline $\mathrm{Ca}\left(\mathrm{cmolc} / \mathrm{dm}^{3}\right)$ & 4,82 & 5,42 & 2,94 & 7,19 & 0,20 & 0,18 \\
\hline $\mathrm{Mg}\left(\mathrm{cmolc} / \mathrm{dm}^{3}\right)$ & 1,39 & 1,37 & 0,60 & 1,10 & 0,31 & $-0,02$ \\
\hline $\mathrm{H}+\mathrm{Al}\left(\mathrm{cmolc} / \mathrm{dm}^{3}\right)$ & 5,05 & 8,25 & 11,39 & 146,24 & 0,33 & 0,23 \\
\hline $\mathrm{SB}\left(\mathrm{cmolc} / \mathrm{dm}^{3}\right)$ & 7,25 & 8,03 & 4,63 & 10,41 & 0,28 & 0,14 \\
\hline CTC $\left(\mathrm{cmolc} / \mathrm{dm}^{3}\right)$ & 12,30 & 16,28 & 16,02 & 156,65 & 0,33 & 0,23 \\
\hline $\mathrm{V}(\%)$ & 43,88 & 44,31 & 29,41 & 47,04 & $-0,28$ & 0,16 \\
\hline $\mathrm{Na}\left(\mathrm{mg} / \mathrm{dm}^{3}\right)$ & 6,47 & 10,55 & 9,42 & 50,95 & $-0,29$ & 0,34 \\
\hline Serapilheira $(\mathrm{cm})$ & 0,10 & 2,40 & 3,70 & 7,90 & 0,05 & 0,45 \\
\hline Umidade gravimétrica (g) & 33,45 & 127,15 & 152,40 & 291,60 & 0,06 & 0,47 \\
\hline
\end{tabular}

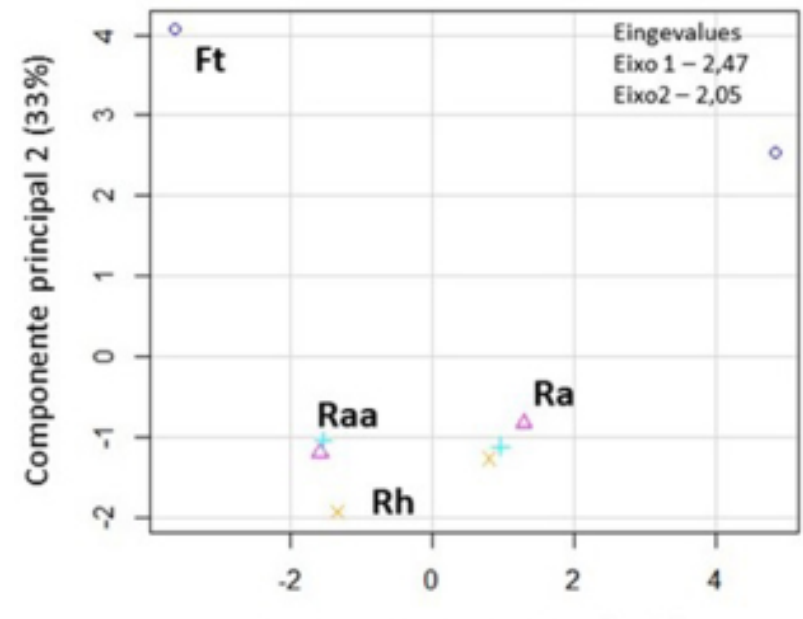

Componente principal $1(46 \%)$

Figura 2. Análise de componentes principais das varáveis edáficas nas quatro formações vegetais de restinga do Parque Estadual Acaraí, São Francisco, Estado de Santa Catarina, Brasil. Restinga Herbácea (Rh, x), Restinga arbustiva (Ra, $\Delta$ ), Restinga arbustivoarbórea $(\mathrm{Raa},+)$, Floresta de transição $\left(\mathrm{Ft},{ }^{\circ}\right)$.

Figure 2. Analysis of the main components of the edaphic variables in the four vegetation formations of the sandbank of the Parque Estadual Acaraí, São Francisco do Sul, Santa Catarina State, Brazil. Herbaceous Restinga (Rh, x), Shrub Restinga (Ra, $\Delta$ ), Shrub-tree Restinga (Raa, + ), Transition Forest $(\mathrm{Ft}, \circ)$.

maiores áreas herbivoradas. Cornelissen e Fernandes (2001) complementam que a área da folha danificada por herbívoros pode ser influenciada pelo teor de água, de açúcares, dureza e teor de nitrogênio das folhas. Isto ocorre porque o nitrogênio é um elemento importante na dieta dos insetos, que apresentam maior preferência pelas folhas ricas nesse elemento (Moran \& Hamilton 1980, Coley \& Barone 1996).

A vegetação herbácea ocupa as primeiras faixas de areia da região pós-praia, local com menor disponibilidade de recursos, além de condições mais restritivas quando comparada às formações seguintes. Gonçalves-Alvim et al. (2011) sugerem que a disponibilidade de nutrientes para plantas em diferentes habitats pode ser um fator determinante na produção de metabólitos secundários. Desta forma, plantas estressadas nutricionalmente, como as de restinga herbácea, devem ter menores concentrações de defesas químicas, tornando-se mais suscetível ao ataque de herbívoros (White 1984).

A expressão de defesas químicas pode variar quando os recursos edáficos diferem em disponibilidade (Ernest 1989), explicando porque a produção de defesas aumentou qualitativamente e quantitativamente no sentido marcontinente. A Floresta de Transição apresentou a maior disponibilidade de metabólitos secundários, o que implica na resistência das plantas às adversidades ambientais (Bussotti et al. 1998) e na defesa contra patógenos e insetos (Marquis et al. 2001). Neste sentido, a presença de metabólitos nos diferentes tecidos da folha, a quantidade de nitrogênio reduzida em relação à restinga herbácea e o menor teor de água podem ter influenciado nas menores taxas de herbivoria encontradas nas espécies desta formação, oposto ao esperado da terceira hipótese deste estudo. Situação similar foi observada em indivíduos de Symphyopappus casarettoi B. L. Rob. (Asteraceae), nos quais os metabólitos secundários foram detectados em mais tecidos nas populações que estão sob melhores condições nutricionais e hídricas (MatildeSilva et al. 2017).

Na restinga arbustivo-arbórea a baixa taxa de herbivoria pode ser devido, principalmente, a uma menor área específica 
foliar e a maior força de perfuração, características observadas em folhas esclerófilas. Plantas submetidas ao estresse hídrico, nutricional e/ou alta intensidade luminosa, tal qual a comunidade em estudo, tendem a investir mais recursos na produção de tecido mecânico, quando comparado ao tecido fotossintético, o que reflete em baixos valores de AEF (Vendramini et al. 2002, Read \& Sanson 2003), resultando em folhas mais espessas e duras, dificultando a ação de insetos herbívoros (Corrêa 2007, Guerra et al. 2010). Na restinga arbustivo-arbórea é onde ocorreram folhas menos nutritivas em quantidade de nitrogênio, podendo influenciar na preferência reduzida pelos herbívoros.

Estrutura edáfica - A variação nutricional e de água nos solos das formações de restinga, especificamente a quantidade de matéria orgânica, $\mathrm{pH}$, serapilheira e umidade, influenciaram na alocação de recursos e produção de defesas nas espécies das comunidades de cada formação. Consequentemente, cada comunidade apresentou padrões morfológicos e nutricionais de acordo com a estruturação edáfica, agindo esta sobre a extensão dos danos causados pelos insetos herbívoros. A disponibilidade de água e nutrientes nas condições do solo são fatores importantes que afetam o crescimento e a qualidade das plantas (White 1984), influenciando indiretamente na preferência pelos insetos (Pais 1998).

A maior alocação de água nos tecidos foliares (suculência) e de nitrogênio nos indivíduos da restinga herbácea foi, provavelmente, influenciada pelo estresse hídrico e menores quantidades de nutrientes disponíveis no solo, implicando na necessidade da planta em armazenar estes recursos. A exposição ao vento, aos borrifos do mar e a deposição de sal existente na restinga delineiam um ambiente de alto estresse (Castanho et al. 2012). Quando submetidas a um forte estresse, as plantas são susceptíveis à degradação de enzimas (Larcher 1986), quando a deterioração enzimática diminui a síntese proteica nas folhas, levando a um aumento da concentração de aminoácidos. Assim, folhas com maior teor de aminoácidos e outros compostos de nitrogênio orgânico livres são mais nutritivas, sendo mais consumidas pelos herbívoros (Price 1991).

Em oposição, a composição edáfica da formação florestal apresentou índices elevados de componentes que indicam um solo mais nutritivo e úmido, pela maior disponibilidade hídrica e quantidade de serapilheira acumulada sobre o solo, implicando em constante disponibilidade de água e ciclagem de nutrientes (Melo Jr. \& Boeger 2016). Devido à disponibilidade de nutrientes, existe a possibilidade de constante produção foliar, assim as plantas investem na produção de tecidos fotossintetizantes em detrimento de tecidos estruturais, o que resulta em folhas mais tenras (maior AEF) (Schoonhoven et al. 1998). O aumento na concentração de nutrientes no solo também influencia na presença de metabólitos secundários nos tecidos foliares (Gershenzon 1984), corroborando os resultados observados nos indivíduos desta formação.

Segundo Neves et al. (2010), as características do solo podem ter efeito bottom-up na atividade de alimentação de herbívoros influenciando, por exemplo, nas características das plantas e alterando a qualidade nutricional dos tecidos vegetais. Neste sentido, pode ser verificado um efeito bottomup para a interação solo-defesa nas espécies estudadas, isto porque a variação da qualidade edáfica entre as formações influenciou diretamente nas estruturas morfoanatômicas e concentração de nitrogênio e compostos secundários nas folhas das espécies em questão e, portanto, tornando-as mais suscetíveis ou resistentes à herbivoria.

\section{Agradecimentos}

Os autores agradecem ao suporte financeiro do Fundo de Amparo à Pesquisa, cedido ao segundo autor, pela Universidade de Região de Joinville e à CAPES pelo concedimento de bolsa ao terceiro autor.

\section{Contribuição dos autores}

Fernanda Vitorio da Silva: Contribuição na coleta de material em campo e processamento em laboratório; Redação do texto.

João Carlos Ferreira de Melo Júnior: Contribuição no delineamento teórico-metodológico da pesquisa; Análise estatística; Revisão do texto; Suporte financeiro.

Maiara Matilde-Silva Contribuição no delineamento teórico-metodológico da pesquisa; Coletas em campo; Revisão do texto.

\section{Conflitos de interesse}

Os autores declaram não haver conflitos de interesse.

\section{Literatura citada}

Agrawal, A.A. \& Fishbein, M. 2006. Plant defense syndromes. Ecology 87: 132-149.

Barthlott, W., Neinhuis, C., Cutler, D., Ditsch, F., Mensel, I., Theisen, I. \& Wilhelm, H. 1998. Classification and terminology of plant epicuticular waxes. Botanical Journal of the Linnean Society 126: 237-260.

Bunrs, E.R. 1971. Method for estimation of tannin in grain sorghum. Agronomy Journal 63: 511-512.

Bussoti, F., Gravano, E., Grossoni, P. \& Tani, C. 1998. Occurrence of tannins in leaves of beech trees (Fagus sylvatica) along an ecological gradient, detected by hystochemical and ultraestrutural analyses. New Phytologist 138: 469-479.

Castanho, C.T. 2012. Facilitação entre plantas e suas implicações para a dinâmica e restauração de restingas. Tese de Doutorado, Universidade de São Paulo, São Paulo.

Coley, P.D. \& Barone J.A. 1996. Herbivory and plant defenses in tropical forests. Annals of Review Ecology and Systematic 27: 305-335.

Coley, P.D., Bryant, J.P. \& Chapin, F.S. 1985. Resource Availability and Plant Antiherbivore defense. Science 230: 895-899.

Cornelissen, T. \& Fernandes, G.W. 2001. Defense, growth, and nutrient allocation in the tropical shrub 
Bauhinia brevipes (Leguminosae). Austral Ecology 26: 246-253.

Corrêa, P. 2007. Defesas foliares em resposta à herbivoria em espécies lenhosas de restinga, Ipojuca-PE. Dissertação de Mestrado, Universidade Federal de Pernambuco, Recife.

Crawley, M.J. 2007. The R book. John Wiley e Sons Ltd., Chichester.

David, R. \& Carde, J.P. 1964. Coloration différentielle des inclusions lipidiques et terpéniques des pseudophylles du Pin maritime au moyen du réactif nadi. Comptes Rendus de l'Académie des Sciences 258: 1338-1340.

Santos, H.G., Almeida, J.A., Oliveira, J.B., Lumbreras, J.F., Anjos, L.H.C., Coelho, M.R., Jacomine, P.K.T., Cunha, T.J.F. \& Oliveira, V.A. 2013. Sistema brasileiro de classificação de solos. 3 ed. Embrapa, Brasília.

Ernest, K.A. 1989. Insect herbivory on a tropical understory tree: effects of leaf age and habitat. Biotropica 21: 194199.

Espírito-Santo, M.M., Neves; F.S., Andrade-Neto, F.R. \& Fernandes, G.W. 2007. Plant architecture and merystem dynamics as the mechanisms determining the diversity of gall-inducing insects. Oecologia 153: 353-364.

Fukami, T., Bezemer, T.M., Mortimer, S.R. \& Putten, W.H. 2005. Species divergence and trait convergence in experimental plant community assembly. Ecology Letters 8: 1283-1290.

Furlan, C.M., Salatino, A. \& Domingos, M. 1999. Leaf content of nitrogen and phenolic compounds and their bearing with the herbivory damage to Tibouchina pulchra Cogn. (Melastomataceae), under the influence of air pollutants from industries of Cubatão, São Paulo. Revista Brasileira de Botânica 22: 317-323.

Gatehouse, J.A. 2002. Plant resistance towards insect herbivores: a dynamic interaction. New Phytologist 156: $145-169$.

Gershenzon, J. 1984. Changes in the levels of plant secondary metabolites and water and nutrient stress. Recent Advances in Phytochemistry 18: 273-320.

Gonçalves-Alvin, S.J., Lana, T.C., Ranieri, B.D. \& Fernandes, G.W. 2011. Test of hypotheses about herbivory and chemical defences of Qualea parviflora (Vochyaceae) in Brazilian Cerrado. Revista Brasil de Botânica 34: 223-230.

Gonçalves-Alvim, S.J., Korndorf, G. \& Fernandes, G.W. 2006. Sclerophylly in Qualea parviflora (Vochysiaceae): Influence of herbivory, mineral nutrients, and water status. Plant Ecolology 187: 153-162.

Guerra, P., Becerra, J. \& Gianoli, E. 2010 Explaining differential herbivory in sun and shade: the case of Aristotelia chilensis saplings. Arthropod-Plant Interactions 4: 229- 235.

Hanley, M.E., Lamont, B.B., Fairbanks, M.M., \& Rafferty, C.M. 2007. Plant structural traits and their role in anti-herbivore defence. Perspectives in Plant Ecology, Evolution and Systematics 8: 157-178.
Hartmann, T. 2004. Plant-derived secondary metabolites as defensive chemicals in herbivorous insects: A case study in chemical ecology. Planta 219: 1-4.

Howe, G.A. \& Schaller, A. 2008. Direct Defenses in Plants and Their Induction by Wounding and Insect Herbivores. In: Schaller, A. Induced plant resistance to herbivory, 2 ed, pp. 7-29.

Johansen, D.A. 1940. Plant microtechnique. New York: McGraw- Hill.

Kabera, J.N., Semana, E., Mussa, A.R. \& He, X. 2014. Plant Secondary Metabolites: Biosynthesis, Classification, Function and Pharmacological Properties. Journal of Pharmacy and Pharmacology 2, pp. 377-392.

Knie, J.L.W. (org.). 2002. Atlas ambiental da região de Joinville: complexo hídrico da Baía da Babitonga. FATMA/GTZ.

Kursar, T.A., Coley, P.D. 2003. Convergence in defense syndromes of young leaves in tropical rainforests. Biochemical Systematics and Ecology, pp. 929-949.

Larcher, W. 1996. Ecofisiologia vegetal. São Paulo: Editora Pedagógica e Universitária.

Leão, Z.M.A.N. \& Dominguez, J.M.L. 2000. Tropical coast of Brazil. Marine Pollution Bulletin 41: 112-122

Loiola, P.P., Silva, I.A., Silva, D.M. \& Batalha, M.A. 2012. Underdispersion of anti-herbivore defence traits and phylogenetic structure of cerrado tree species at fine spatial scale. Journal of Vegetation Science 23: 1095-1104.

Matilde-Silva, M., Boeger M.R.T., Melo Jr., J.C.F., Santos, B.F.S. 2017.Antiherbivory defense mechanisms along an environmental gradient in restinga. Acta Botanica Brasilica. 31(4): 583-596.

Marques, E.S.A., Price, P.W. \& Cobb, N.S. 2000. Resource abundance and insect herbivore diversity on woody fabaceous desert plants. Environmental Entomology 29: 696-703.

Mauricio, R. 2000. Natural selection and the joint evolution of tolerance and resistance as plant defenses. Evolutionary Ecology 14: 491-507.

Marquis, R.J., Diniz, I.R. \& Morais, H.C. 2001. Patterns and correlates of interspecific variation in foliar insect herbivory and pathogen attack in Brazilian Cerrado. Journal of Tropical Ecology 17: 127-148.

Melo Jr., J.C.F. \& Boeger, M.R.T. 2015. Riqueza, estrutura e interações edáficas em um gradiente de restinga do Parque Estadual do Acaraí, Estado de Santa Catarina, Brasil. Hoehnea 42: 207-232.

Moran., N. \& Hamilton, W.D. 1980. Low nutritive quality as defence against herbivores. Journal of Theoretical Biology 86: 247-254.

Neves F.S., Araújo, L.S. Espírito-Santo, M.M., Fagundes, M., Fernandes, G.W., Sanchez-Azofeifa, G.A. \& Quesada, M. 2010. Canopy herbivory and insect herbivore diversity in a dry forest-Savanna transition in Brazil. Biotropica 42: 112-118.

Pais, M.P. 1998. Valor nutritivo e investimento em defesas em folhas de Didymopanax viscosum E. March. e sua relação com a herbivoria em três fisionomias do cerrado. 
Dissertação de Mestrado, Universidade de São Paulo, São Paulo.

Paiva, J.G.A., Fank-de-Carvalho, S.M., Magalhães, M.P. \& Graciano-Ribeiro, D. 2006. Verniz vitral incolor 500: uma alternativa de meio de montagem economicamente viável. Acta Botanica Brasilica 20: 257-264.

Price, P.W.1991. The Plant Vigor Hypothesis and Herbivore Attack. Oikos 62(2): 244-251.

Probio. 2003. Áreas prioritárias para a conservação, utilização sustentável e repartição de benefícios da biodiversidade brasileira. MMA.

Read, J. \& Sanson, G.D. 2003. Characterizing sclerophylly: The mechanical properties of a diverse range of leaf types. New Phytologist 160: 81-99.

Root, R.B. 1972. Organization of a plant-arthropod association in simple and diverse habitats: the fauna of collards (Brassica oleracea). Ecological Monographs 43: $95-124$

Sampaio, D., Souza, V.C., Oliveira, A.A., Paul-Souza, J. \& Rodrigues, R.R. 2005. Árvores da restinga: guia ilustrado para a identificação de espécies da Ilha do Cardoso. São Paulo: Neotrópica.

Scarano, F.R. 2002. Structure, function and floristic relationships of plant communities in stressful Habitats to the Brazilian Atlantic rainforest. Annals of Botany 90: 517-524.

Scarano, F.R., Duarte, F.L.S., Ribeiro, M., Rodrigues, T., Barcellose, P.J.F., Franco, M.B., Brulfertj, C., Eléense, D. \& Lüttge, U. 2001. Four sites with contrasting environmental stress in southeastern Brazil: relations of species, life form 31 diversity, and geographical distribution to ecophysiological parameters. Botanical Journal of the Linnean Society 136: 345-364.

Schoonhoven L.M., Jermy T. \& Von Loon, J.J.A. 1998. Insect-plant biology: from physiology to evolution. London: Chapman Hall.

Silva, D.M. \& Batalha, M.A. 2011. Defense syndromes against herbivory in a Cerrado plant community. Plant Ecology 212: 181-193.

Tedesco, M.J., Gianello, C. Bissani, C.A. Bohnen, H. \& Volkweiss, S.J. 1995. Análise de solo, plantas e outros materiais. 2 ed. Universidade Federal do Rio Grande do Sul, Porto Alegre.

Turner, I.M. Sclerophylly: Primarily protective. 1994. Functional Ecology 8: 669-675.

Vendramini, F., Díaz, S., Gurvich, D.E., Wilson, P.J., Thompson, K. \& Hodgson, J.G. 2002. Leaf traits as indicators of resource-use strategy in floras with succulent species. New Phytologist 154: 147-157.

White, T.C. 1984. The abundance of invertebrate herbivores in relation to the availability of nitrogen in stressed food plants. Oecologia 63: 93-105.

Yasuhara, T. \& Nokihara, K. 2001. High-throughput analysis of total nitrogen content that replaces the classic Kjeldahl method. Journal of Agricultural and Food Chemistry 49: 4581-4583.

Recebido: 04/03/2021

Aceito: 16/11/2021

Editor Associado: Natália Macedo Ivanauskas 